\title{
21 Including participants who cannot communicate in research on ageing?
}

\author{
Linda J. Garcia and Louise Bélanger-Hardy
}

This chapter focuses on participant selection in research, in particular the involvement of older adults with communication challenges. With the ageing of the population and the increased prevalence of chronic health conditions such as stroke and dementia, a significant number of older individuals are left with difficulties that impair their ability to communicate or to engage in conversations. This has a profound impact on how researchers include them in research. Indeed, limited communication poses challenges not only for obtaining informed consent but also for engagement in surveys, interviews, focus groups or even in responding to surveys. The exclusion of this group of older adults has a wide impact because policymakers are relying on studies from which the most affected individuals have either been excluded or not properly included. In the chapter, the authors explore the ethico-legal and practical challenges facing researchers who consider the inclusion of this group of older adults in their protocols. A number of best practices are offered to researchers, institutional review boards and granting agencies with the aim of better responding to the need for increased participation while ensuring protection from harm and exploitation.

\section{Introduction}

Our complex ability to communicate and use language is one of humankind's most distinguishing characteristics. From as early as our days of living in caves, communication, through pictures, speech and, later, writing, has been an integral part of our lives. It connects us with others, it allows us to do the things that give us meaning and it helps us find safe places to live. Therefore, it is not surprising to find that communicative abilities affect quality of life. In no demographic group is the impact of communication disorders more evident than in the older population, where many health conditions such as stroke or dementia are associated with resulting problems in exchanging information. Communication is complex and how it affects lives depends greatly on the elements of communication that are needed to respond to the individual's needs. Even at the societal level, the emphasis we have placed on pictures, reading, writing or speaking has changed over time as well as what we have preferred as a means of expression from poems to emojis (McCulloch, 2019). Consequently, someone who cannot write would likely choose conversation over email and someone with limitations in expressing themselves verbally might do better with writing. The effect of living with a communication difficulty is not homogeneous. Hence, learning more about the lived experiences of individuals who struggle to communicate and learning about how they can adapt to societal demands is of paramount importance to public policy. Unfortunately, because of the inherent difficulties associated with including 
people with communication disorders in research protocols, we know less about the impact of communication disorders as compared to other functional limitations. For instance, how does a stroke with resulting language impairment differ in terms of impact on quality of life from a stroke that does not result in language impairment? How do these outcomes compare with the quality of life of those who live with arthritis? Or, more specifically, how do disorders affecting verbal expression differentially impact daily life as opposed to disorders with comprehension, or reading, or articulation, or word finding, or even decreased voice volume? An older individual who must speak in public may need greater voice volume than one who does not. Living with a condition such as Parkinson's disease, the consequence of which might be lowered voice volume, can be more devastating to one person than to someone else (Miller et al., 2006). Understanding the true impact of health conditions which affect communication is essential.

More knowledge from research will inform policymakers to reduce the number of obstacles in accessing public services or navigating urban and rural spaces. Easing communication barriers might also help older individuals connect with loved ones and help reduce social isolation. Unfortunately, much of the data that might inform public policies is lacking because of the inherent difficulties in conducting research with participants who struggle to take part in these studies. It may not be surprising that this group is often excluded. Research protocols involve being able to respond to questions given orally or in writing or being able to exchange information through conversation or through groups. The purpose of this chapter is to highlight the importance of including older individuals with communication disorders in research protocols, to explore the ethico-legal and practical challenges faced by researchers and to offer some solutions and stimulation for debate to help address these challenges.

\section{Background}

Some of the most common health conditions in older adults cause disorders of communication. A stroke might cause a problem with speech or language, Parkinson's disease might affect one's voice and cognitive problems like dementia might affect one's ability to maintain conversations (Brookshire and McNeil, 2015). Individuals with communication problems may have concomitant cognitive problems affecting judgment and decision-making such as in moderate to advanced dementia. However, this is not the case for all individuals with communication problems. A great many others, whose communication problem is caused by a single stroke, such as in aphasia, may be fully capable of giving consent and making decisions, provided some effort or adaptation is made to bypass the communication difficulty (Brady, Fredrick and Williams, 2013). Unfortunately, many researchers, policymakers and members of civil society fail to acknowledge this difference. As early as 1995, Kagan made reference to aphasia as a mask of an individual's true competence (1995). Unless the interlocutor attempts to lift the mask by facilitating communication and revealing the underlying competence, judgments researchers make about their capacities may remain erroneous. The older adult may thus be falsely judged as being incompetent and incapable of making decisions and reliance on an authorized third party might be inappropriate for these participants. Such perceptions may result in exclusion from research studies. Misperceptions can result in significant social isolation, impingement on life-long learning, inability to participate in community life or in maintaining interpersonal relationships without this needing to be the case (Chapey et al., 2000). Clearly it is important for clinicians and the community 
at large to understand these distinctions. But, as well, the research community on ageing must seriously consider including these individuals in their studies.

The need for society to adapt to those with functional limitations is not new. In 2001, the World Health Organization launched its International Classification of Functioning, Disability and Health (ICF) (WHO, 2018). The ICF was developed as a classification system serving as a common language worldwide to document the impact of various health conditions on body systems, functional limitations and social participation. Part of the classification also identified environmental factors such as physical design features (e.g., wheelchair ramps), assistive technological aids and other factors such as natural environmental changes like climate, changes in social support, government policies and programs. Through research and conceptual frameworks clarifying the relationship between level of impairment, environmental factors and the resulting situation of handicap, it is possible to arrive at policies that modify environmental factors and make life better for those with functional limitations. This reasoning, for instance, has led employers to understand how wheelchair users can contribute significantly to their workplace if only they were provided with elevator access or ramps. Likewise, researchers wishing to include wheelchair users in their protocols must make sure that their testing labs are accessible. Impairment with communication, however, presents special challenges to being included in research protocols since much of the methods used to enquire about lived experiences requires language. As a consequence, the recognition of the potential of individuals with communication disorders is less understood and their value less recognized for lack of providing "communication ramps" (Kagan, 1995). Surely the lived experiences of those who survive a stroke and retain their ability to communicate are different from those who become noncommunicative. Measures of quality of life after stroke, based on data taken from participants who could talk cannot be generalized to participants who cannot speak. Our understanding of experiences based on data gathered from non-aphasic stroke survivors will necessarily bias how policymakers and the general public view the post-stroke experience (Mazaux et al., 2013; Ellis and Peach, 2017). Policies might focus primarily on the physical adaptations needed by those with hemiplegia or other physical limitations, in part because people with aphasia were not included in early studies. In addition, it is hard to have a voice and make your challenges known when communication is difficult. Individuals with aphasia have exceptional challenges advocating for themselves in public fora. Research can go a long way in making their needs and experiences known to others.

It is important for researchers to understand the consequences of exclusion. Lessons can be learned from other populations where a subgroup was once excluded or not singled out from research results. One such example comes from studies on the presenting symptoms of cardiovascular disease (Bucholz and Krumholz, 2015). Women were dying of cardiovascular disease in greater numbers than men. As the results from studies on men were generalized to women, it remained unrecognized that women presented symptoms that were different from men. Many early public awareness campaigns of warning signs were therefore based on initial studies where there occurred a gender imbalance in study participants (Dougherty, 2011). Following careful examination of the subpopulation of women, it became clear that a new awareness campaign was necessary and one could not rely on the earlier studies to educate the public (Brown, 2015). Likewise, in Canada, where the French-speaking population finds itself in a minority situation in most of the country, it has become important to understand how services, which are mandated by law, are not necessarily designed to respond to the needs of the francophone population. Even though they seem to respond to the general need of the majority English speaking population, this is not 
always the case for the French speaking population (Schofield and Gauthier, 2007). The recognition of this difference has led some institutional review boards to require that researchers indicate the reasons for excluding any linguistic population group. In a country such as Canada, which prides itself on its respect of diversity, questions about inclusion are important for individuals of various faiths, genders, socio-economic status, ethnic origins or languages. Some research ethics boards now ask researchers to justify if they have included participants who identify with different genders and whether these groups will be analyzed differently. This is also the case for some important marginalized groups in Canada such as members of the aboriginal population (SSHRC, 2018). Not including them may lead policymakers and clinicians to design policies and interventions that are ill adapted or simply unhelpful to the target population. Exclusion, whether intentional or not, can have grave consequences and the same can be said about excluding older participants who have difficulty communicating.

Prioritizing policies and programs based on the skills and adaptations needed to make the world a more communication-friendly place for older adults with communication problems should be an important social objective. Even in age-friendly and dementia-friendly community recommendations, there is reference to communication as one of the key parameters (WHO, 2015). Yet, there is very little that speaks to how one might adapt differently to someone who has challenges understanding versus someone who has challenges expressing themselves. The research community would benefit from a more detailed consideration of these subgroups in research on ageing.

Being convinced of the importance of including participants with communication disorders is only the first step. Understandably, researchers might find accommodating their protocols to this population difficult. If the research community is ready to adapt how it does research with this section of our population, then there should be some level of understanding of what communication is and how it can be impaired.

\section{What is communication?}

It is difficult to obtain statistics on the incidence and prevalence of communication disorders. The National Institute on Deafness and Other Communication Disorders in the United States estimates that between six and eight million Americans have some form of language impairment and approximately 7.5 million have trouble using their voices (NIDCD, 2016). Generally, it is agreed that about one-third of stroke survivors have aphasia but the rates are hard to obtain and can vary from 18-38 percent, precisely because of the consistency in reporting this disorder (Ellis et al., 2018). There is no clear or reliable way to know what the prevalence of disorders of communication is with the older population. The Australian Bureau of Statistics concluded that of its 1.8 million older Australians living with a disability in 2015, 42 percent had a functional limitation with communication (Australian Bureau of Statistics, n.d.). It is also estimated that 70-90 percent of individuals with Parkinson's disease have problems with their speech and voice (Lirani-Silva et al., 2015). These statistics are not negligible and when we add the diagnosis of dementia to the mix, the number of individuals with difficulties in communication skyrockets. With the ageing of the population worldwide, the need to understand the impact of these disorders from a communication standpoint and for comparison to those who can communicate is undeniable.

Communication is a complex process that involves much more than speaking and understanding. It involves an intricate process of encoding and decoding through verbal and 
non-verbal means, analysis and interpretation of content, adjustment of interpretation of rapidly incoming information and thoughts, and elaborate methods of expression as sounds, words, sentences and conversations are created through a physiological web of coordinated muscles and timing, or translated to written forms or signs.

\section{Researchers' need to adapt}

How researchers adapt to individuals who struggle with any of these processes can have profound impact on the relationship they have with these participants and the results they obtain. This means that any challenges in communication in individual A can impact how the researcher will interpret and interact with that individual. Not only can the message be affected by the communication disorder itself, but the researcher may be distracted by these difficulties and forget to seek the underlying message. As different communication disorders should be differentially considered for research, so too must they be studied separately as we consider the impact on people's lives. In addition to factors related to the speaker, the hearer and the message, extraneous factors can cloud the communication process (DeVito, 2016). For instance, there is the "method" through which the message is transmitted (i.e., phone, etc.), the interference as the message is being transmitted (e.g., sudden noise), the encoding and decoding processes and abilities (e.g., reading non-verbal cues), the context (e.g., a job versus participation in a research study) as well as the feedback that is given and perceived throughout the exchange. All these factors can influence the outcome of the exchange even in people without communication disorders, but their impact might be amplified when communication is difficult. Adapting to these participants can go a long way in obtaining relevant and useful data and obtaining informed consent.

One classic example is the difference between aphasia, a language difficulty resulting from stroke, and cognitive language abilities resulting from dementia (Worrall and Frattali, 2000). A large number of individuals with aphasia from a single stroke typically do not have judgment impaired because of their stroke and they are fully capable of giving consent and participating given that a protocol is adapted to their needs (Brady et al., 2011). Dementia typically affects other cognitive parameters such as memory and judgment and can hinder someone's ability to maintain a conversation or to conceptually understand what is required. Adaptation to this type of disorder is different from aphasia. People in the early stages of dementia might be able to give consent but because of the additional cognitive impairments, attention needs to be given to the contribution of the communication difficulties to the overall cognitive picture. Similarly, someone with dysarthria or speech and voice disorders will have other communication challenges that people with dementia or aphasia may not have (Brady, et al., 2011). They might be able to circumvent the verbal expression of the message by using written channels or communication aids and may have absolutely no difficulty understanding what is said to them. Hence, lumping all communication disorders into one category is unfair and unethical. Some individuals who could fully give consent and participate in research might be denied access to research as participants simply because they have a communication disorder, without any consideration on the part of the researcher to adapt to their condition.

In considering the role of researchers, a crucial point is that the ability to consent to research cannot be based on diagnosis alone. Of course, in some cases, the stroke or dementia will affect competence. In others, less so and individuals will be able to give informed consent. This is not an all or none phenomenon. Therefore, determining capacity is key to engaging individuals who have difficulty communicating into research protocols. 
Consequently, in this chapter, we have chosen to be somewhat fluid about the distinction between dementia and aphasia, in order to focus on the notion of capacity. The following section addresses the need to clarify the legal and ethical responsibilities arising within this context.

\section{Inclusion in research - human rights and ethico-legal perspectives}

Several international instruments are relevant to a discussion on the ethical and legal dimensions of including older adults with communication challenges in research protocols. For instance, the United Nations Principles for Older Persons (UNPOP; United Nations, General Assembly, 1991) promotes the independence, participation, care, self-fulfillment and dignity of older citizens. More specifically, in terms of participation, UNPOP provides that older persons "should remain integrated in society, participate actively in the formulation and implementation of policies that directly affect their well-being and share their knowledge and skills with younger generations" (UNPOP, article 7). By highlighting social integration and active participation, UNPOP emphasizes the importance of incorporating the input of older persons in all facets of life. This includes the possibility of involvement in research, especially if the focus is on older individuals' health and well-being. A second relevant international instrument is the Declaration of Helsinki (DoH), adopted in 1964 by the World Medical Association (World Medical Association, 2013). The DoH provides, as one of its general principles, that "[g]roups that are underrepresented in medical research should be provided appropriate access to participation in research" (General Principle 13). As well, the American National Institutes of Health (NIH), has adopted the Inclusion Across the Lifespan policy, taking effect as of 25 January 2019. The age-related inclusion policy applies to all NIH conducted or supported research involving human subjects and requires that "individuals of all ages, including children ... and older adults, must be included in all human subjects research, conducted or supported by the NIH, unless there are scientific or ethical reasons not to include them" (National Institutes of Health, 2017). In Canada, the Tri-Council Policy Statement (TCPS2), the guideline for the ethical conduct of research involving humans in that country, provides at Article 4.1 that researchers shall not exclude individuals from the opportunity to participate in research on the basis of age unless there is a valid reason for the exclusion (TCPS2, 2018). As can be seen, these international and national instruments clearly support inclusion of older persons in research, especially when they come from populations that are not adequately represented such as persons with communication challenges.

Despite the principles outlined in these international instruments, in the context of clinical and non-clinical research participation, the traditional approach has been to lean toward great caution as far as older adults and people with functional limitations are concerned. Several studies have shown that both researchers and research ethics committees will routinely exclude participants who are perceived as "vulnerable" (Finnigan and O'Donoghue, 2019). This includes older adults in general (Jensen et al., 2019), but also children, people with dementia and adults lacking capacity. However, in recent years, there have been many calls for fuller participation of these so-called vulnerable populations (Cascio and Racine, 2019), and this has led to intense debates about how best to protect these participants and, at the same time, respond to the moral demand to conduct research related to the underrepresented groups to which they belong (Shepherd, 2016; Helmchen et al., 2014).

Research involving humans may expose participants to physical, emotional, privacy or other risks. Therefore, both legally and ethically, participants must provide voluntary 
and informed consent. Based on autonomy and self-determination, informed consent insures that participants make their own decisions grounded on their own values and goals. Informed consent is both an ethical and legal requirement to participation in research. Several elements are involved, namely disclosure, comprehension, voluntary choice and authorization (TCPS2, 2018, art. 3.2). Of great importance is the fact that informed consent goes beyond simply obtaining a person's agreement to participate. Rather, it is a process where, through the dialog with the researcher, potential participants assess risks, benefits, alternatives and other relevant information such as withdrawal, dissemination of findings, protection of confidential data etc. in order to make their decision (TCPS2, 2018, art. 3.3)

Crucially, in order to participate in the informed consent process, the participant must have the required capacity. Therein lies one of the main challenges when considering research with older adults coping with disorders affecting communication. Indeed, their difficulty in communicating in a conventional manner does not necessarily mean they lack the required capacity to provide informed consent to a research project.

In both the legal and ethical context, capacity is defined through a functional approach. In the legal realm, capacity is defined in relation to care and treatment and involves the ability to (a) understand relevant information and (b) appreciate the potential consequences of the treatment (e.g., Health Care Consent Act, 1996 (HCCA), section 4(1)). In many jurisdictions, this two-prong test has been adapted and applied to the research context (Vaishnav and Chiong, 2018, p. 540). A good example is the Canadian TCPS2 where decision-making capacity is defined in terms of understanding information presented about a research project and appreciating the consequences of the decision to participate or not (TCPS2, 2018, art. 3.9).

From a legal standpoint, adults are presumed to have the capacity to consent to treatment (e.g., HCCA, section 4(2); Mental Capacity Act, 2005, section 1(2)) and by extension to research participation. This being said, recognizing that, for some individuals, difficulties in communicating will be linked to health conditions impacting the capacity to make informed decisions is essential. If researchers suspect that an adult potential participant does not have the required capacity, they must carry out a capacity assessment. If capacity is lacking, specific protection mechanisms (recourse to a third party for instance) will become essential. This is where the role of substitute decision-makers or surrogates becomes very important. Nonetheless, the argument made here is that researchers and Institutional Review Boards (IRBs) should not assume that, because a person has communication challenges, they necessarily lack capacity to provide informed consent.

Capacity is challenging to assess and requires the researcher's professional judgment. The fact that capacity may vary depending on the context, or on the moment at which it is assessed, is one complicating factor. While disorders common in older adults such as stroke or dementia affect individuals' abilities, communication skills and possibly judgment differently, the impact of these disorders may change drastically over the course of the trajectory of the illness or condition. In some cases, the communication disorder may lessen or even subside while, in others, it will get worse.

But, perhaps more importantly, protocols, policies and laws often provide little guidance about when and how to assess capacity (Dalpé et al., 2019, p. 7). Indeed, Vaishnav and Chiong $(2018$, p. 540) emphasize that "there is no clear legal standard specifically tailored to determining capacity to consent to research". In such a context, it is perhaps unsurprising that researchers and ethics committees will err on the side of caution when they perceive that a participant may belong to a population seen as "vulnerable". 
This being said, there are validated instruments, such as the MacArthur Competence Assessment Tool for Clinical Research, on which researchers have relied to assess research decisional capacity. This tool is based on structured interviews that purport to measure understanding, appreciation, reasoning and expression of choice (Vaishnav and Chiong, 2018 , p. 541). However, when dealing with older adults with communication disorders these instruments are not necessarily ideal (Jeste et al., 2007). Indeed, the researcher's task is to insure that the information conveyed is received and understood and that the person has the tools to convey their willingness to participate. Evidently, having difficulties expressing oneself poses real challenges. As mentioned previously, participants with communication disorders may inherently have their competence "masked" by their disorder and may struggle in making it known to others that they understand the information being given to them and that they appreciate the consequences of the decision to participate. In these cases, the researchers grapple with the decision as to whether they need to go to a substitute decision-maker or not. For example, in a study on randomized clinical trials for neurovascular emergencies, Rose and Kasner note that researchers "subjectively estimate" the capacity to provide informed consent and decide, on their own, if surrogate consent is called for (2011, p. 4). Therefore, there is a need for further development of capacity assessment tools for non-communicative potential participants. Some studies have started to explore better adapted tools, often condition specific (e.g., Seaman et al., 2015).

In some cases, participants with communication disorders may be seen as "vulnerable" in the research context. Vulnerability is not easy to define. In fact, there is disagreement over its meaning and application in research ethics. In their survey of major international and national research ethics policies, Bracken-Roche et al. (2017) found only a few definitions in their sample. One of them comes from Canada's TCPS2 2018 where the following definition is suggested:

A diminished ability to fully safeguard one's own interests in the context of a specific research project. This may be caused by limited decision-making capacity or limited access to social goods, such as rights, opportunities and power. Individuals or groups may experience vulnerability to different degrees and at different times, depending on their circumstances.

(Glossary "vulnerability")

The TCPS2 2018 addresses directly participant's vulnerability and research in Article 4.7 and states that participants should not be inappropriately included or automatically excluded from participation in research on the basis of their circumstances. This chapter is based on the core principles at the heart of the TCPS2 2018, namely Respect for Persons, Concern for Welfare and Justice as well as on the notion of Equitable Distribution of Research Benefits. This last element entails that participants in research projects may draw "direct benefits" (better health for instance), or "indirect benefits" (advancement of knowledge improving conditions for the group to which the participant belongs) from their participation (TCPS2, 2018, art. 47). The crucial point is that vulnerable groups are entitled to these benefits. The challenge is to attain this objective without compromising a potential participant's safety. Finally, Bracken-Roche et al. (2017) point out the growing literature arguing that vulnerability is relative in nature and flows from power imbalances between potential participants and researchers and IRBs. They suggest that recognizing this phenomenon could stimulate a closer examination of the notion of vulnerability by IRBs and researchers who may consequently be more attentive to the importance of empowerment 
and the promotion of agency when dealing with "so-called" vulnerable adults (BrackenRoche et al., 2017, p. 16).

One of the common safeguards when seeking the participation of a potential participant whose capacity to decide may be compromised is to rely on an authorized third party who has the legal authority to make decisions on the participant's behalf. Reliance on such third parties is not without challenges, however. First, while a number of national and international instruments provide for the use of a legally authorized substitute when necessary (e.g., DoH), others such as Ontario, Canada's largest province, expressly provide that such representatives cannot be relied on in the context of consent to research (HCCA, section 6(1)) (see Thorogood et al., 2018, p. 87). Second, some concern is expressed in the literature about the authorized third party's ability to properly identify the participant's preferences or safeguard his or her interests (e.g., Finnegan and O'Donoghue, 2019, p. 67). In the case of an older adult struggling with communication, the surrogate may face additional challenges in ascertaining the wishes, intentions and preferences of the participant - even if capacity is present. Exploring fully this question is not possible within this chapter but the importance of further reflection and research on the matter is undeniable if one is to promote more active participation in research by older adults with communication challenges.

As can be seen in the discussion above, international human rights instruments provide a strong basis for the inclusion of older adults in research protocols. However, researchers and ethics committees are hesitant to include participants who they perceive as lacking the capacity to provide informed consent because of age or of functional limitations, including communication challenges. While improvement of capacity assessment tools may assist researchers, a number of other measures may be envisaged as well. Researchers must provide a "safe space" for participants to fully take part in research projects. Importantly, researchers who rely mainly on a diagnosis to determine if a person will be included in a research project will not be in a position to recognize if the potential participant's ability to understand the information provided, or to appreciate the consequences of participating and the choices offered. The next section of this chapter highlights some of the suggested strategies that might be adopted to conform to the principles of good research when including older individuals who have communication disorders.

\section{Best practices for inclusion of older individuals with communication disorders in research}

There are yet no universally accepted guidelines regarding the inclusion of people with communication disorders in research on ageing. What follows are strategies to consider for improving how researchers interact with participants whose communication is a challenge. As mentioned previously, one of the fundamental concerns is that judgment not be based solely on diagnosis but rather assume some sensitivity to the nature of the communication issues, regardless of diagnosis. Two of the most common primary sources of literature guiding researchers on how to interact with older adults who have difficulties making their needs known are studies on aphasia and on dementia. The case of dementia is interesting because it is now recognized that individuals with this condition can and should be active participants in research and efforts should be made to obtain informed consent as often as possible (Brady et al., 2013). While these individuals may also have some difficulties with decision-making and appreciating the choices in front of them, some attempt should be made to reduce the communication barriers. What follows are suggestions for researchers, 
institutional review boards, publishers and granting agencies and for communities of older adults who live with communication challenges. It is beyond the scope of this chapter to offer an exhaustive summary of techniques for involving older adults with communication challenges in research. The reader is encouraged to consult the very rich literature on the lived experiences of aphasia for examples. Our purpose is to stimulate dialog amongst researchers who would normally exclude such participants and to the organizations that support fair and ethical research on this issue.

\section{For researchers}

1 Be open. Assume capacity but be sensitive to possible vulnerabilities. Regardless of diagnosis, the researcher should assume capacity and be cognizant that the communication difficulties exhibited by the participant may indeed be masking an underlying competence to participate in the research protocol (Dalemans et al., 2009). In other words, researchers should be open to this possibility and be ready to adapt their protocols to give potential participants a fair chance of being included. Recognizing that including these individuals in research will help shed light on the nature of the disorders as well as the impact it might have on those who live with these challenges is crucial. By including them in larger studies that are not focused on the communication aspect itself, researchers will be contributing to knowledge of the differential impact of communication vis-à-vis other functional limitations.

2 Adapt your recruitment strategies. As part of a report on a research summit on dementia care, organized by the Office of the Assistant Secretary for Planning and Evaluation in the US Department of Health and Human Services, Lepore and colleagues (2017) have outlined some cautionary advice and some useful suggestions for recruiting people with dementia into research. There is a sensitivity to person centeredness that is also relevant to older adults with communication disorders in general. One advice is to be aware of the danger of relying on gatekeepers based on the common belief and bias that individuals with communication challenges cannot participate in research. In many studies, recruitment will rely on healthcare staff and sometimes family members to identify potential participants. In fact, Bartlett, Milne and Croucher (2018) specifically suggest that nurses be part of recruitment strategies. While this is indeed a useful approach, the participation bias noted above is also common in these gatekeepers. Some health professionals may not have the time to assess the potential participants in some depth and may have forgotten or not known that abilities are context dependent. This means that while some potential participants might appear to be non-functional in their milieu, in the right context and with the right support, they could very well participate in a research project. As a result, some gatekeepers may underestimate the participant's competence and consequently omit to refer them to the researcher for inclusion. Lepore and colleagues (2017) suggest that one way of diversifying recruitment strategies is to use modalities that do not rely on gatekeepers but, rather, connect directly with the potential participants themselves. These might include online recruitment through support groups or by engaging advocacy groups and health charities such as aphasia associations or dementia societies. Another strategy is to make sure that recruitment documents are accessible from a communication standpoint, such as aphasia-friendly documentation (Howe et al., 2004). In some cases, it is important to recognize that information may be easier to understand through written language for some, and through non-verbal means (e.g., pictographic) for others. Recruitment 
strategies, therefore, need to use multiple modalities if they are to reach the persons with communication disorders themselves.

3 View obtaining informed consent as a complex process. Obtaining informed consent for research is far from straightforward and, as mentioned above, the desire to respect choice and decision-making is challenged by a researcher's ability to determine capacity. Penn and colleagues (2009) rightly point out that obtaining informed consent is a process, involving more than the simple signature on a legal document. Every effort should be made to provide a research context where people with communication challenges can have the time to understand what is involved and have the support to communicate their choices. Penn and colleagues provide an excellent breakdown of all the components that are often part of informed consent, from understanding the rationale of the project to knowing whom to contact. In their study they videotaped, transcribed and analyzed in detail the interactions between participants with aphasia and researchers while informed consent was being sought for a clinical trial. Participants were later interviewed and asked, amongst other things, whether the study had met their expectations. While the authors included just a small number of participants, the in-depth analysis highlights the need to make sure that information is well received and processed from the outset. How informed consent is obtained in this population is crucial to inclusion and adherence to principles of ethical research. Yet, in the same paper, Penn and colleagues reviewed and found that only 41 out of 458 existing studies that included people with aphasia had mentioned obtaining informed consent, and only a few actually described the process. The quality and ability of researchers to obtain consent with this population is partly dependent on the researcher's ability to communicate with participants and some useful suggestions are summarized below. Minimally, obtaining informed consent should be done in a face-to-face encounter in a quiet environment, and consideration should be given to the use of pictogram-based consent forms made available by entities such as the Aphasia Institute. Finally, because it is difficult to know what the person has understood, some authors (Jensen et al., 2019, p. 329) suggest a re-evaluation of informed consent a few hours after the initial decision to include has been made.

4 Rely on a legally authorized third party as a last resort. All efforts must be made to include the participant before seeking consent from a legally authorized third party (Lepore et al., 2017). As is often noted, the surrogate's perceptions of lived experiences are not always synchronized with the participant's perceptions and care should be taken not to rush to the conclusion that the potential participant lacks capacity or is unable to take part in the research (Mendyk et al., 2015). As discussed above, however, there is a need for further development of tools to assess capacity of older adults with communication challenges.

5 Adjust data collection. The communication strategies mentioned above are not only useful for obtaining informed consent but also for all aspects of a research protocol that involves understanding or transmitting information. Because much of what we do as researchers involves communication, it is also appropriate to adjust our style to insure that we obtain the best data possible from participants who have difficulties understanding or expressing themselves. Contrary to individuals who may just need time to say what they want, as may be the case with someone who has problems with articulation, individuals with more advanced dementia might need frequent reminders and facilitators, such as visual support material, to contribute to the interview (Smeets and Moser, 2012, p. 12). In the case of questionnaires, if not time constrained, the 
participant can respond at his or her leisure. In the case of focus groups, others can help to provide context, but the researcher must be careful to give the participant who cannot express himself or herself easily, the time to respond. Sometimes the communication strategies outlined below can help the participant understand or get his or her point across, even in focus group or interview formats.

6 Diversify communication strategies. Whether the researcher is recruiting, obtaining consent, judging for assent or dissent, or engaging in communication-based protocols, some knowledge, training or adaptation is necessary if individuals with difficulties communicating are to be included in a protocol. What follows are some suggestions for researchers to consider. Readers are strongly urged to consult the literature on the training of communication partners and supportive conversation. A seminal article by Kagan and colleagues (2001) leads to a body of research on the topic.

a Since the researcher will likely not have a detailed evaluation of the potential participant's communication challenges, access to multiple ways of making the information understood would be useful. Aids such as photos, written material, videos, objects and pictograms could facilitate communication. Similarly, the participant should have access to various ways of communicating with the researcher such as writing or drawing instruments and paper.

b The key to successful communication is flexibility with the strategies that are used. If something is not working in terms of comprehension or if the researcher struggles to understand what the participant is saying, another strategy from the communication toolkit should be tried. Importantly, reliance solely on oral communication should be avoided as some participants will need one or more different modes of communication at different times in order to reveal what they know or want to say.

c Researchers should insure that the environment is quiet so that the person who has the communication problem can focus on the task at hand.

d Researchers should insure that there are no sensory barriers to communication. This means that hearing aids and reading glasses should be in place if necessary.

e The atmosphere should foster a feeling of trust and patience. Periods of silence may mean a need for extra processing time. Research staff are encouraged to treat participants as competent adults, regardless of their status.

f In order to make sure that messages have been correctly understood by the participant, research staff could ask alternate specific questions to verify apparent understanding. This will prevent the participant from simply repeating what the research staff has said and will increase the chances that the participant has processed the information.

g As well, research staff can paraphrase and repeat back what they have understood. This gives participants a chance to correct or confirm the message they were trying to convey.

$\mathrm{h}$ Researchers might consider hiring a trained language expert to facilitate with protocols and obtaining consent (McCormick et al., 2017).

7 Develop materials for research. Some of the reasons leading to the exclusion of individuals with communication disorders include the lack of standardized tools for inclusion and of modified consent forms, as well as the absence of research staff able to adapt to the needs of this population for any given protocol. Research protocols adapted for people with communication difficulties are therefore needed and must be 
tested for validity and reliability. Researchers who include older adults with dementia or stroke in their studies will interact with individuals with communication difficulties on a regular basis. The need for sharing and evaluating the impact of strategies is pressing indeed.

\section{For institutional review boards}

Institutional review boards have strict guidelines regarding research protocols with "vulnerable" populations. In trying to protect these populations, these guidelines may in fact be doing a disservice to many groups and individuals alike. As discussed previously in this chapter, participants might be denied access to research when they can indeed participate provided there is some degree of adaptation. Indeed, some studies show that IRBs are at times "overly focussed on legal risk and not always hearing the voice of older adult research participants" (Pachana et al., 2015, p. 705). Researchers who would like to be granted more flexibility in their approaches are restricted by IRBs. More recently Jensen and collaborators (2019) have offered some interesting guidelines which may be considered by IRBs. Because diagnosis is not sufficient to determine capacity, these authors suggest that the assessment be based on the following four elements. We have added questions which IRBs might ask investigators to consider and answer prior to granting ethical approval.

1 Understanding. What measures has the researcher put in place in order to insure that the potential participant has understood the information essential to make an informed decision?

2 Appreciation. What measures has the researcher put in place to assess whether the participant is able to determine the effect and consequences of his or her choice?

3 Reasoning. What measures has the researcher put in place in order to ascertain the participant's comprehension of the rationality and reasonableness of the request, within the current context? Can the participant judge that the request is reasonable?

4 Choice. What measures has the researcher put in place to insure that the participant can communicate his/her choice?

Before a participant is excluded from a project, Jensen and colleagues propose strategies for determining capacity. These suggestions are made within the context of dementia but the strategy seems quite respectful of participants' inclusion in the decision process and should be considered for potential participants with communication challenges. For instance, because their population base is dementia, the decision to accept a person in a study first includes scores on a cognitive test. However, the decision to include or exclude is not limited to these scores and is further refined based on the level of risk associated with the study. Jensen and colleagues propose to include a level of flexibility in situations where the possibility of a participant wrongly agreeing to take part in a study creates a minimal risk of harm. There are further adjustments for all levels of cognitive scores where measures for evaluating assent or dissent must be included in the protocol, and these measures must include interpretation of non-verbal communication. Furthermore, Jensen and colleagues recommend the presence of individuals who are trusted by the participant to support the decision-making process. While these individuals are not there to act as proxies, they can provide support and help create a safe space for communication. The strategies proposed by these authors for individuals with dementia are respectful of 
participants' (in)ability to communicate and make their needs known. They also recognize that the needs may change over time. Regardless of whether IRBs decide to adopt such strategies, they could, at a minimum, encourage researchers to engage participants who might at first seem unable to participate.

\section{For granting agencies and publishers}

More and more, researchers are indeed asked to make an effort to include the voices of the population of interest into their studies. In the area of ageing, this will increasingly include individuals whose voices are difficult to accommodate. In order to do this, while adhering to the ethical principles of autonomy, beneficence and justice, granting agencies and publishers will need to accept that a certain level of flexibility is required when assessing research protocols in which communication challenged individuals are considered as participants. For instance, agencies could check if a protocol includes more time for recruitment and for execution of the research. Granting agencies may need to consider budgets that help researchers overcome the costs associated with increased use of materials or specialized research staff. They may need, as well, to consider the standardization of pictogrambased assessment tools that facilitate communication in research. Publications should incorporate some flexibility in accepting details about how informed consent was obtained and what efforts were made to be inclusive. This will allow readers the chance to properly evaluate the meaningfulness of the results. Adhering to principles of inclusion involves time and money. As researchers are urged to adopt programs that are properly adapted to populations who have communication challenges, they may need extra funds and a more prominent place in publications to insure that this population gets the place it deserves in scientific inquiry.

\section{For older individuals with communication disorders}

The individuals that have the most to lose from not being included in research are, of course, the older individuals with communication disorders. Advocates of older adults might promote efforts for inclusion of all individuals whose voices must be heard. Armed with the knowledge that some older individuals are actually excluded from research without scientific basis, advocates might urge the research community to adapt research protocols, to revisit assumptions about capacity and its link to communication, to avoid exclusive reliance on diagnosis to determine possible inclusion and to consider the use of less traditional tools to explore informed consent.

\section{Conclusion}

The number of older adults who live with communication challenges will likely increase in the foreseeable future given the anticipated growth of the older adult population worldwide. Older adults who face challenges with communication could contribute to the advancement of knowledge regarding the impact of their conditions whether they draw direct benefits from participation or not. However, currently, while there is some opening toward the inclusion of so-called "vulnerable populations" in research, there is still a tendency by IRBs and the research community to exclude individuals with communication challenges from protocols, all the more if they are older. This is perhaps understandable to a certain point given that informed consent, the key ethical and legal safeguard for research 
participants, is dependent on the communication of information and the researcher/ participant interaction. As well, communication is essential for recruitment, written or oral participation, focus groups and interview. However, there is a need to revisit this state of affairs and to consider how best to respond to the need for increased participation while ensuring that participants are not exposed to harm or exploitation.

As discussed above, current key international instruments support the right to participate in research and outline quite clearly the need of non-discrimination and respect of individual autonomy - even as people grow older and/or deal with challenging health conditions. This is an excellent backdrop for the research community to embrace inclusion and to reflect on how best to proceed. In the chapter, we have identified the need to focus on capacity and the development of tools to assess more efficiently participants' ability to understand the information they are given and to appreciate the consequences of the choices they make. We have noted as well new trends in the understanding of vulnerability as a concept having the potential to empower people otherwise left behind. We have challenged researchers to be sensitive to the subtleties of communication in general, regardless of diagnosis, and to consider a number of measures to augment the cohort of older adults with communication issues in their research projects. A number of suggestions have been made to the research community to enhance the participation of these individuals.

Recently, interest for the inclusion of traditional "vulnerable populations" in protocols has intensified worldwide. While the literature on dementia has developed particularly quickly, studies on older adults with reduced communication abilities have been less common. This chapter has highlighted the need for research communities to increase their contribution to the understanding of the true impact of living with communication challenges. This quest for better knowledge will only be possible if participation in research is facilitated. This is, after all, what is expected of us all within our legal and ethical contexts.

\section{References}

Australian Bureau of Statistics (n.d.). Australians living with communication disability. Available at: www.abs.gov.au/ausstats/abs@.nsf/Latestproducts/4430.0Main+Features872015?opendocument\& tabname $=$ Summary\&prodno $=4430.0 \&$ issue $=2015 \&$ num $=\&$ view $+=\#$ OlderAustralians $\quad[$ Accessed 15 September 2019].

Bartlett, R., Milne, R. and Croucher, R. (2018). Strategies to improve recruitment of people with dementia to research studies. Dementia, May. doi:10.1177/1471301217748503.

Bracken-Roche, D., Bell, E., Macdonald, M.E. and Racine, E. (2017). The concept of "vulnerability" in research ethics: an in-depth analysis of policies and guidelines. Health Research Policy and Systems, 15(8), 1-18.

Brady, M.C., Clark, A.M., Dickson, S., Paton, G. and Barbour, R.S. (2011). The impact of strokerelated dysarthria on social participation and implications for rehabilitation. Disability \& Rehabilitation, 33(3), 219-128. doi:10.3109/09638288.2010.517897.

Brady, M.C., Fredrick, A. and Williams, B. (2013). People with aphasia: capacity to consent, research participation and intervention inequalities. International Journal of Stroke, 8(3), 193-196. doi:10.1111/j.1747-4949.2012.00900.x.

Brookshire, R.H. and McNeil, M.R. (2015). Introduction to neurogenic communication disorders. St Louis, MO: Elsevier. Available at: https://books.google.ca/books?hl=en\&lr=\&id=wM9sBQAAQBAJ $\&$ oi $=$ fnd\&pg $=$ PP1\&dq=neurogenic + communication+disorders\&ots=vYAIL198n_\&sig=2fP5I-cF9H EmJp4uaUSh2ranSzk\#v=onepage \&q=neurogenic + communication+disorders\&f=false [Accessed 14 September 2019]. 
Brown, N. (2015). How the American Heart Association helped change women's heart health. Circulation. Cardiovascular Quality and Outcomes, 8(2), Suppl. 1, S60-62. doi:10.1161/CIRCOUT COMES.115.001734.

Bucholz, E.M. and Krumholz, H.M. (2015). Women in clinical research: what we need for progress. Circulation. Cardiovascular Quality and Outcomes, 8(2), Suppl. 1, S1-3. doi:10.1161/CIRCOUT COMES.115.001756.

Canadian Institutes of Health Research, Natural Sciences and Engineering Research Council of Canada, and Social Sciences and Humanities Research Council (TCPS2) (2018). Tri-council policy statement: ethical conduct for research involving humans. December. Available at: www.pre. ethics.gc.ca/eng/policy-politique_tcps2-eptc2_2018.html [Accessed 14 September 2019].

Cascio, A. and Racine, E. (2019). Research involving participants with cognitive disability and differences. Oxford: Oxford University Press.

Chapey, R., Duchan, J.F., Elman, R.J., Garcia, L.J. and Kagan, A. (2000). Life participation approach to aphasia: a statement of values for the future. ASHA Leader, 5(3), 4-6. doi:10.1044/leader. FTR.05032000.4.

Dalemans, R., Wade, D.T., van den Heuvel, W.J.A. and de Witte, L.P. (2009). Facilitating the participation of people with aphasia in research: a description of strategies. Clinical Rehabilitation, 23(10), 948-959. doi:10.1177/0269215509337197.

Dalpé, G., Thorogood, A. and Knoppers, B.M. (2019). A tale of two capacities: including children and decisionally vulnerable adults in biomedical research. Frontiers in Genetics, 10(289). doi: 10.3389/fgene.2019.00289.

DeVito, J.A. (2016). The interpersonal communication book. Harlow, UK: Pearson. Available at: www.vitalsource.com/educators/products/interpersonal-communication-book-the-joseph-a-devitov9780133913835?term=9780133913835 [Accessed 15 September 2019].

Dougherty, A.H. (2011). Gender balance in cardiovascular research: importance to women's health. Texas Heart Institute Journal, 38(2), 148-150. www.ncbi.nlm.nih.gov/pubmed/21494523.

Ellis, C. and Peach, R.K. (2017). Life satisfaction and aphasia: an integrative review with recommendations for future research. Aphasiology, 31(6), 631-642. doi:10.1080/02687038.2016.1154500.

Ellis, C., Hardy, R.Y., Lindrooth, R.C. and Peach, R.K. (2018). Rate of aphasia among stroke patients discharged from hospitals in the United States. Aphasiology, 32(9), 1075-1086. doi:10.1080/0268 7038.2017.1385052.

Finnigan, M. and O'Donoghue, B. (2019). Rethinking vulnerable groups in clinical research. Irish Journal of Psychological Medicine, 36, 63-71.

Health Care Consent Act, 1996, S.O. 1996, c. 2, Sch. A.

Helmchen, H., Hoppu, K., Stock, G., Thiele, F., Vitiello, B. and Weimann, A. (2014). From exclusion to inclusion: improving clinical research in vulnerable populations. Berlin: BerlinBranderburg Academy of Sciences and Humanities.

Howe, T.J., Worrall, L.E. and Hickson, L.M.H. (2004). What is an aphasia-friendly environment? Aphasiology, 18(11), 1015-1017.

Jensen, J.S., Reiter-Theil, S., Celio, D.A., Jakob, M., Vach, W. and Saxer, F.J. (2019). Handling of informed consent and patient inclusion in research with geriatric trauma patients: a matter of protection or disrespect? Clinical Interventions in Ageing, 14, 321-334. doi:10.2147/CIA.S191751.

Jeste, D.V. Palmer, B.W., Appelbaum, P.S., Golshan, S., Glorioso, D., Dunn, L.B., Kim, K., Meeks, T. and Kraemer, H.C. (2007). A new brief instrument for assessing decisional capacity for clinical research. Archives of General Psychiatry, 64(8), 966-974.

Kagan, A. (1995). Revealing the competence of aphasic adults through conversation: a challenge to health professionals. Topics in Stroke Rehabilitation, 2(1), 15-28. doi:10.1080/10749357.1995.11754051.

Kagan, A. Supported conversation for adults with aphasia: why \& how? Selected Slides. p. 12.

Kagan, A., Black, S.E., Duchan, J.F., Simmons-Mackie, N. and Square, P. (2001) Training volunteers as Conversation Partners Using "Supported Conversation for Adults With Aphasia" (SCA): a controlled trial. Journal of Speech, Language, and Hearing Research, 44(3), 624-638. doi:10.1044/1092-4388(2001/051). 
Lepore, M., Shuman, S.B., Wiener, J.M. and Gould, E. (2017). Challenges in involving people with dementia as study participants in research on care and services. Research Summit on Dementia Care. Available at: https://aspe.hhs.gov/system/files/pdf/256696/Session 5 Background.pdf [Accessed 26 June 2019].

Lirani-Silva, C., Mourão, L.F. and Gobbi, L.T. (2015). Dysarthria and quality of life in neurologically healthy elderly and patients with Parkinson's disease. CoDAS, 27(3), 248-254. doi:10.1590/ 2317-1782/20152014083.

McCormick, M., Bose, A. and Marinis, T. (2017). Decision-making capacity in aphasia: SLT's contribution in England. Aphasiology, 31(11), 1344-1358. doi:10.1080/02687038.2017.1355441.

McCulloch, G. (2019). Because internet: understanding the new rules of language. New York: Riverhead Books.

Mazaux, J., Lagadec, T., de Sèze, M.P., Zongo, D., Asselineau, J., Douce, E., Trias, J., Delair, M.F. and Darrigrand, B. (2013). Communication activity in stroke patients with aphasia. Journal of Rehabilitation Medicine, 45(4), 341-346. doi:10.2340/16501977-1122.

Mendyk, A-M., Labreuche, J., Henon, H., Girot, M., Cordinnier, C., Duhamel, A., Leys, D. and Bordet, R. (2015). Which factors influence the resort to surrogate consent in stroke trials, and what are the patient outcomes in this context? BMC Medical Ethics, 16. doi:10.1186/s12910-015-0018-8.

Mental Capacity Act 2005 (UK), 2005, c. 9.

Miller, N., Noble, E., Jones, D. and Burn, D. (2006). Life with communication changes in Parkinson's disease. Age and Ageing, 35(3), 235-239. doi:10.1093/ageing/afj053.

National Institutes of Health (2017). NIH policy and guidelines on the inclusion of individuals across the lifespan as participants in research involving human subjects. Notice Number: NOT-OD-18116. Bethesda, MD: National Institutes of Health.

NIDCD (2016). Statistics on voice, speech, and language. National Institute on Deafness and Other Communication Disorders. Available at: www.nided.nih.gov/health/statistics/statistics-voicespeech-and-language [Accessed 14 September 2019].

Pachana, N.A., Liddle, J., Peel, N.M., Beattie, E., Juang, C. and Knight, B.G. (2015). Can we do better? Researchers' experiences with ethical review boards on projects with later life as a focus. Journal of Alzheimer's Disease, 43, 701-707.

Penn, C., Frankel, T., Watermeyer, J. and Müller, M. (2009). Informed consent and aphasia: evidence of pitfalls in the process. Aphasiology, 23(1), 3-32. doi:10.1080/02687030701521786.

Rose, D.Z. and Kasner, S.E. (2011). Informed consent: the rate-limiting step in acute stroke trials. Frontier in Neurology, 2(65). doi: 10.3389/fneur.2011.00065.

Seaman, J.B., Terhorst, L., Gentry, A., Hunsaker, A., Parker, L.S. and Lingler, J.H. (2015). Psychometric properties of decisional capacity screening tool for individuals contemplating participation in Alzheimer's disease research. Journal of Alzheimer's Disease, 46(1), 1-9. doi:10.3233/JAD-142559.

Schofield, A. and Gauthier, H. (2007). The society "Santé en Français": a successful Canadian model for partnership. Education for Health: Change in Learning and Practice, 20(2). www.scopus.com/ inward/record.url?eid=2-s2.0-34948878050\&partnerID=40.

Shepherd, V. (2016). Research involving adults lacking capacity to consent: the impact of research regulation on "evidence-biased" medicine. BMC Medical Ethics, 17. doi:10.1186/s12910-016-0138-9.

Smeets, H. and Moser, A. (2016). A guide to ensure client participation in research on people who suffer from dementia. ZUYD. Available at: www.innovatiesindezorg.eu/files/5214/8853/9827/ DEF_Client_participation_in_clients_with_dementia.pdf [Accessed 26 June 2019].

SSHRC (2018). Toward a successful shared future for Canada: research insights from the knowledge systems, experiences and aspirations of First Nations, Inuit and Métis peoples. Social Sciences and Humanities Research Council. Available at: www.sshrc-crsh.gc.ca/society-societe/communitycommunite/ifca-iac/03-aboriginal_peoples_in_Canada_report-les_peuples_autochtones_en_ Canada_rapport-eng.pdf [Accessed 26 June 2019].

Thorogood, A., Dalpe, G., McLauchlan, D. and Knoppers, B. (2018). Canadian consent and capacity regulation: undermining dementia research and human rights? McGill Journal of Law and Health, 12(1), 67-121. 
United Nations, General Assembly (1991). United Nations principles for older persons. Resolution 46/91, 16 December. Available at: www.ohchr.org/Documents/ProfessionalInterest/olderpersons. pdf [Accessed 1 July 2019].

United Nations (2014). Committee on the right of persons with disabilities: general comment no. 1. Available at: https://documents-dds-ny.un.org/doc/UNDOC/GEN/G14/031/20/PDF/G1403120. pdf?OpenElement [Accessed 3 July 2019].

Vaishnav, N.H. and Chiong, W. (2018). Informed consent for the human research subject with a neurological disorder. Seminars in Neurology, 38(5), 539-547. doi:10.1055/s-0038-1668077.

WHO (2015). Global age-friendly cities: a guide. World Health Organization.

WHO (2018). International classification of functioning, disability and health (ICF). World Health Organization. Available at: www.who.int/classifications/icf/en/ [Accessed 17 July 2019].

World Medical Association (2013). Declaration of Helsinki: ethical principles for medical research involving human subjects. 18th WMA General Assembly, June 1964, amended by the 64th WMA General Assembly, October 2013.

Worrall, L. and Frattali, C. (2000). Neurogenic communication disorders: a functional approach. New York: Thieme. 